\title{
Enhanced speed and sensitivity in the cultural diagnosis of pulmonary tuberculosis with a continuous automated mycobacterial liquid culture (CAMLiC) system
}

\author{
J. G. MAGEE, R. FREEMAN and A. BARRETT \\ PHLS Regional Centre for Mycobacteriology, Newcastle Public Health Laboratory, Westgate Road, \\ Newcastle upon Tyne NE4 GBE
}

\begin{abstract}
A total of 2800 sputum samples referred for mycobacterial investigation was examined by both continuous automated mycobacterial liquid culture (CAMLiC) and conventional Loewenstein-Jensen culture (LJC). The CAMLiC system was more sensitive than LJC, detecting $188(98.4 \%)$ of 191 of all mycobacteria found by one or both methods compared to $150-162(78.5-84.8 \%)$ found by LJC (the range for LJC takes into account all potential 'missed positives' due to contamination). Figures for Mycobacterium tuberculosis complex (MTBC) organisms specifically were $133(98.4 \%)$ of 135 for CAMLiC and 115-122 (85.2-90.4\%) for LJC. Detectable growth of MTBC organisms in CAMLiC occurred at a mean of 13.4 days after inoculation (range 3-32; SD 6.49; mode 8 days); $65.4 \%$ of such isolates were detected within 14 days and $87.2 \%$ within 21 days. In 73 instances the MTBC status of the isolate was defined by gene probe on the day of growth detection. Sufficient biomass for valid gene probe assay was always present. The speed, sensitivity and labour-sparing technology (no manual intervention is necessary before identification or discard) of CAMLiC make it possible for many laboratories to approach the Centers for Disease and Prevention (CDC) standard for culture and identification in mycobacteriology without resort to direct DNA detection techniques and at a much lower cost.
\end{abstract}

\section{Introduction}

The Centers for Disease Control and Prevention (CDC: Atlanta, GA, USA) has formulated recommendations for the diagnosis and control of tuberculosis [1]. These include laboratory standards for detection by smear, culture and identification (to species level) and antibiotic susceptibility determination. The isolation and identification standard has been taken to be 10-14 days. This figure is usually interpreted as a mean or average value. It has been argued that this stringent standard will be unachievable without resort to direct nucleic acid amplification methods applied to clinical material. However, it has also been demonstrated that the cost of following this route may be unsupportable [2], notwithstanding some remaining problems in sensitivity and specificity for such techniques [3]. In a recent clarification [4], CDC have stated the isolation and identification standard to be 14-21 days. However,

Received 10 April 1997; revised version accepted 27 Oct. 1997.

Corresponding author: Professor R. Freeman. this less stringent figure is not taken to be a mean value and it is expected that all but a few organisms will be detected and identified within 21 days.

Increases in both the sensitivity and speed of cultural diagnosis of tuberculosis have been claimed for the application of semi-automated mycobacterial liquid culture (SAMLiC) systems [5]. Although more expensive than conventional culture, these methods are significantly cheaper for examination of clinical material than direct nucleic acid detection methods. However, only two of 10 laboratories using a commercially available radiometric SAMLiC system, supplemented by direct nucleic acid probe (gene probe) identification of the culture, met the earlier CDC standard [2]. The reasons cited for the failure of the other eight similarly equipped laboratories to attain that standard were staffing patterns, scheduling of culture examination and specimen processing and the timing of gene probe-based identification. Five of the laboratories reached the revised CDC standard, but three of the remainder fell well short. The adoption of a continuous automated mycobacterial liquid culture 
(CAMLiC) system in which specimens, once inoculated, undergo continuous monitoring for growth detection (in contrast to a semi-automated system in which batches of specimens must be manually removed from incubation for examination daily) can be expected to permit more laboratories to adopt this technology successfully and, thereby, meet the revised CDC criteria, and even approach the earlier, more stringent, isolation and identification standard.

A large evaluation of a CAMLiC system was performed in the diagnosis of pulmonary tuberculosis. The study compared critically the CAMLiC results with those of conventional solid Loewenstein-Jensen cultures (LJC) on the same clinical material.

\section{Materials and methods}

A total of 2800 sputum samples referred for mycobacterial examination was investigated over an 18month period. In all instances, the specimens were first digested and homogenised with dithiothreitol (Sputasol, Oxoid, Basingstoke) which was added in equal volume to the sample and left to stand for $15 \mathrm{~min}$. Decontamination was by the addition of $\mathrm{NaOH} 4 \%$ and standing the mixture at ambient temperature for 25 min with intermittent agitation. Neutralisation was achieved with $0.5 \mathrm{~N}$ sulphuric acid, with phenol red as indicator. The resultant material was then centrifuged ( $3000 \mathrm{~g}$ for $15 \mathrm{~min}$ ) and the pellet was resuspended in $5 \mathrm{ml}$ of the supernate. A vial of CAMLiC medium (modified Middlebrook $7 \mathrm{H} 9$ broth medium with antibiotic supplement and growth factors; Organon-Teknika Ltd, Cambridge) was inoculated with $0.5 \mathrm{ml}$ of the concentrate and placed in the incubation chamber of the MB Bact CAMLiC system (Organon-Teknika). Similar volumes $(0.5 \mathrm{ml})$ were used to inoculate two Loewenstein-Jensen slopes, one glycerol-based and the other pyruvate-based, which were incubated under standard conditions for up to 9 weeks. LJ slope cultures were examined at weekly intervals for the presence of mycobacteria. The CAMLiC system continuously monitors $\mathrm{CO}_{2}$ production by an infra-red detection system. Once the $\mathrm{CO}_{2}$ production exceeds a pre-set level, the system signals growth detection and the vial is removed for further examination. All samples were examined microscopically for the presence of acid-fast bacilli by a fluorescent staining method [6].

When mycobacteria were detected in one or other culture system they were identified by standard methods. In addition, a gene probe specific to the Mycobacterium tuberculosis complex (MTBC) (GeneProbe Inc, San Diego, CA, USA) was used on selected occasions, including the identification of first isolates from previously undiagnosed mycobacterial infection or in circumstances in which there were clinical grounds for urgency (for instance, suspected recurrence, relapse or re-isolation during therapy). When non-mycobacterial growth was detected in either system (CAMLiC or LJC), the specimen was decontaminated again and re-inoculated. Detection intervals were always calculated with reference to the date of re-inoculation. However, if mycobacterial growth was already detected in the comparison sample (used in the alternative system) repeat-decontamination was not attempted and the result was recorded as 'contaminated'.

At the end of the 6-week incubation period recommended by the manufacturer, CAMLiC vials which had not flagged positive were removed from the machine. They were not examined further, but were not discarded until the corresponding LJC was discarded after a further 3 weeks.

\section{Results}

The overall performance of the two systems being compared is shown in Table 1. Mycobacteria were isolated from $191(6.8 \%)$ of 2800 sputum specimens, the vast majority (135 of 191: 70.7\%) being species within the MTBC. Set against the total numbers of mycobacteria detected by one or other method, the sensitivity of the CAMLiC system for all mycobacteria was $188(98.4 \%)$ of 191 , compared with $150(78.5 \%)$ of 191 for LJC. Even if it is assumed that all

Table 1. The isolation rates of 191 different mycobacteria from 2800 sputum samples by CAMLiC compared with LJC

\begin{tabular}{|c|c|c|c|c|}
\hline \multirow{3}{*}{$\begin{array}{l}\text { Mycobacterial } \\
\text { species }\end{array}$} & \multicolumn{4}{|c|}{ Number detected/total isolates $(\%)$ by } \\
\hline & \multicolumn{2}{|c|}{ CAMLiC method } & \multicolumn{2}{|c|}{ LJC } \\
\hline & $A^{*}$ & $\mathbf{B}^{\dagger}$ & $A^{*}$ & $\mathrm{~B}^{\dagger}$ \\
\hline M. tuberculosis & $133 / 135(98.4 \%)$ & $133 / 135(98.4 \%)$ & $115 / 135(85.2 \%)$ & $122 / 135(90.4 \%)$ \\
\hline M. avium & $15 / 15 \quad(100 \%)$ & $15 / 15 \quad(100 \%)$ & $10 / 15 \quad(66 \%)$ & $13 / 15 \quad(87 \%)$ \\
\hline M. malmoense & $18 / 18 \quad(100 \%)$ & $(100 \%)$ & $(50 \%)$ & $(56 \%)$ \\
\hline M. kansasii & $18 / 19 \quad(95 \%)$ & $(100 \%)$ & $(79 \%)$ & $(79 \%)$ \\
\hline${ }^{\ddagger}$ Others & $4 / 4$ & $4 / 4$ & $3 / 4$ & $4 / 4$ \\
\hline All species & $188 / 191(98.4 \%)$ & $189 / 191(98.9 \%)$ & $150 / 191(78.5 \%)$ & $162 / 191(84.8 \%)$ \\
\hline
\end{tabular}

*Assumes that all mycobacteria-containing contaminated cultures were negative by this method.

${ }^{\dagger}$ Assumes that all mycobacteria-containing contaminated cultures were positive by this method.

${ }^{\dagger}$ M. fortuitum (2) M. szulgai (2). 
contaminated cultures (predominantly in the LJC system) in which the comparator culture was positive were 'missed positive', the figures become $98.9 \%$ versus $84.8 \%$.

For MTBC isolates, CAMLiC detected 133 (98.4\%) of 135 , against $115(85.2 \%)$ for LJC. Assuming once again that any LJC contaminated cultures paired with CAMLiC positive cultures were actually positive gives figures of $133(98.4 \%)$ for CAMLiC and 122 (90.4\%) for LJC.

Sensitivity rates for CAMLiC for the isolation of $M$. avium-intracellulare complex (MAC), M. malmoense and $M$. kansasii were $100 \%, 100 \%$ and $94.7 \%$, compared with $66.7 \%, 50 \%$ and $78.9 \%$, respectively, for LJC. These latter figures improve a little if contamination is discounted and contaminated cultures are assumed to be missed positives, but the rate in each category is still much lower than the CAMLiC figure.

Fig. 1 shows the intervals between inoculation of a processed specimen on to the CAMLiC system and the detection of growth by the system for MTBC isolates. The range of these intervals was 3-32 days, with a mean of 13.38 days (SD 6.49), a mode of 8 days and a median of 12 days. Eighty-seven (65.4\%) of the $133 \mathrm{MTBC}$ isolates were detected within 14 days of inoculation and $116(87.2 \%)$ within 21 days. Fig. 1 also shows that $131(98.5 \%)$ of the 133 MTBC isolates were detected within 28 days. Isolates of three other species (MAC, M. malmoense and M. kansasii) were detected at intervals which spanned the 28 days (Fig. 2), necessitating species identification on each occasion. The small numbers of other species (two isolates each of $M$. fortuitum and $M$. szulgai) are not shown in the figures but are recorded in Table 1. Conventional identification was performed on all 191 isolates. Gene probe assessment of MTBC status (+ or -) was also performed on 73 isolates on the day of detection. In 70 instances sufficient biomass was present in uncentrifuged fluid from the CAMLiC vial. Centrifugation was required in the other three instances. In all 73 instances the gene probe results were identical to those of the conventional tests. Fourteen of the 135 specimens from which MTBC organisms were isolated were negative in the auramine smear examination. The interval between inoculation and detection and identification in the CAMLiC system for this subset of samples ranged from 13 to 28 days (mean 21.6 days; SD 3.6 days).

Fig. 3 compares the cumulative weekly isolation rates of $M$. tuberculosis from 2800 sputum specimens by the two methods. Although the intervals between inoculation and detection for the CAMLiC system were measured in days they have been plotted in quanta of weeks to allow direct comparison with the LJC results. It is clear that the CAMLiC culture system detected more isolates of $M$. tuberculosis than the LJC system and where M. tuberculosis was detected by both methods, CAMLiC achieved detection significantly quicker. Fig. 3 also demonstrates that almost all of both these advantages are achieved within 4 weeks of inoculation and that prolongation of CAMLiC incubation beyond this point confers little further advantage.

Contamination with non-mycobacterial organisms occurred in $152(5.4 \%)$ of the 2800 specimens and 12 of these instances were in specimens from which mycobacterial species were eventually isolated by one or other method. Growth (due to contaminants) was detected in all 12 such specimens within 7 days in the CAMLiC system and successful repeat decontamination was achieved in 11 of these instances, followed by re-inoculation and detection of mycobacterial growth in a mean of a further 12.3 days (range 6-18). As contamination in the LJC system was rarely detected within 7 days, the presence of mycobacteria in the CAMLiC system was usually known by the time that LJC contamination was evident and re-decontamination was not attempted for LJC. In one instance contamination was detected in the CAMLiC system, but re-decontamination was unsuccessful. However, the LJC sample of the same specimen yielded $M$. kansasii.

\section{Discussion}

The results of the present study show that the CAMLiC system enhanced both the sensitivity and speed of isolation of all mycobacterial species and, in particular MTBC organisms, from sputum samples, confirming and extending our preliminary report [7]. At least 11 and probably up to $18 \mathrm{MTBC}$ organisms were isolated in the CAMLiC system which would not have been detected by conventional LJC. Furthermore, MAC, $M$. malmoense and $M$. kansasii were also isolated by the CAMLiC system which were not isolated on LJC (Table 1) indicating that the CAMLiC system is more sensitive than LJC.

The enhanced speed with which mycobacterial growth was detected with the CAMLiC system was also impressive and this was particularly important for MTBC organisms. Laboratories that use the CAMLiC system tested here can expect to be able to satisfy the CDC revised standard, in that growth was detected at a mean interval of 13.4 days and sufficient biomass was available at the time of detection to permit successful gene probe identification on the same day [2]. In practice, the distribution of positive results is skewed (the mode interval was 8 days) and the system detected two out of three MTBC isolates within 14 days and nine out of 10 within 21 days. Both of the significant advantages of the CAMLiC system over conventional culture are realised in the first 30 days. 


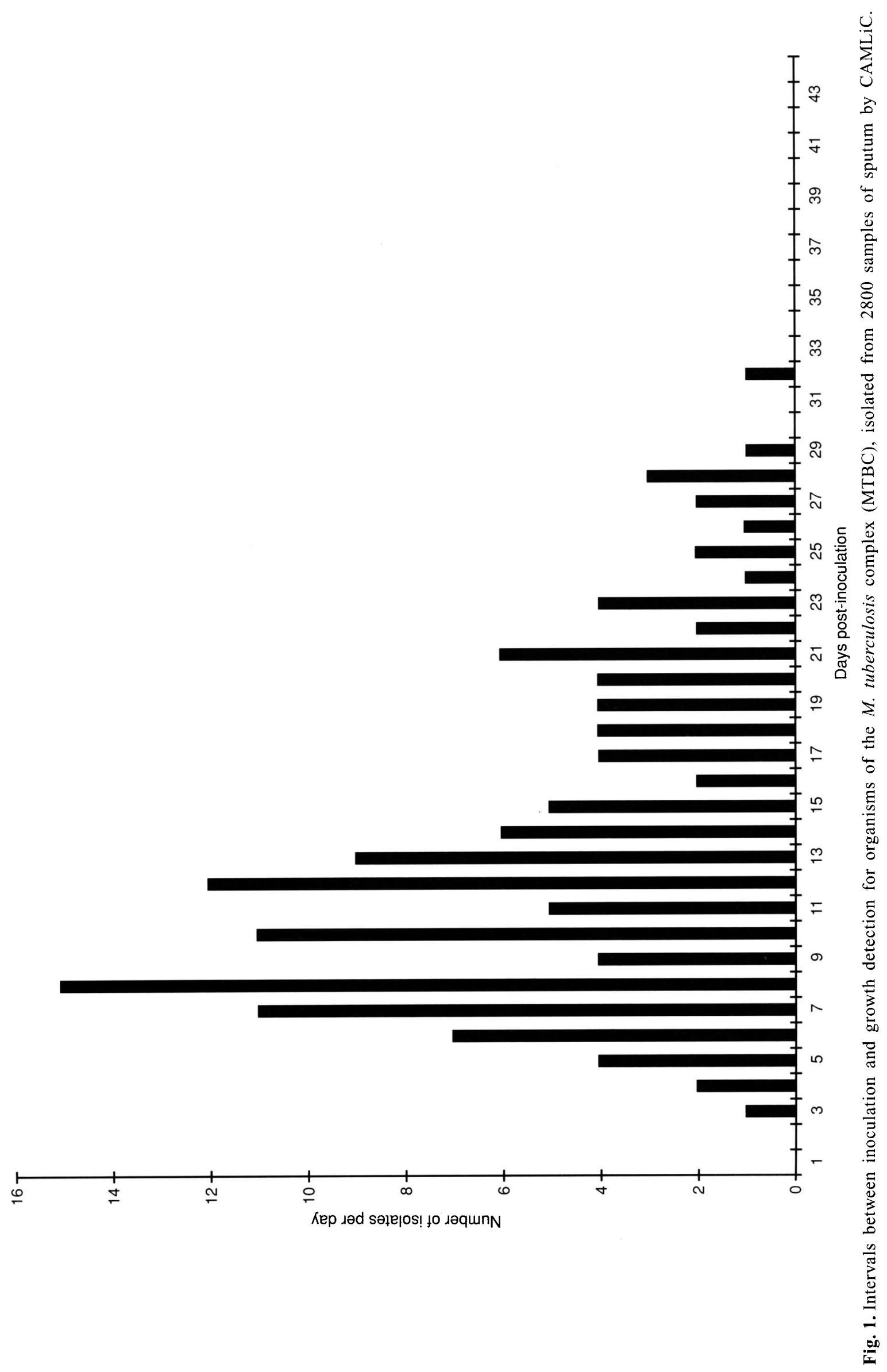




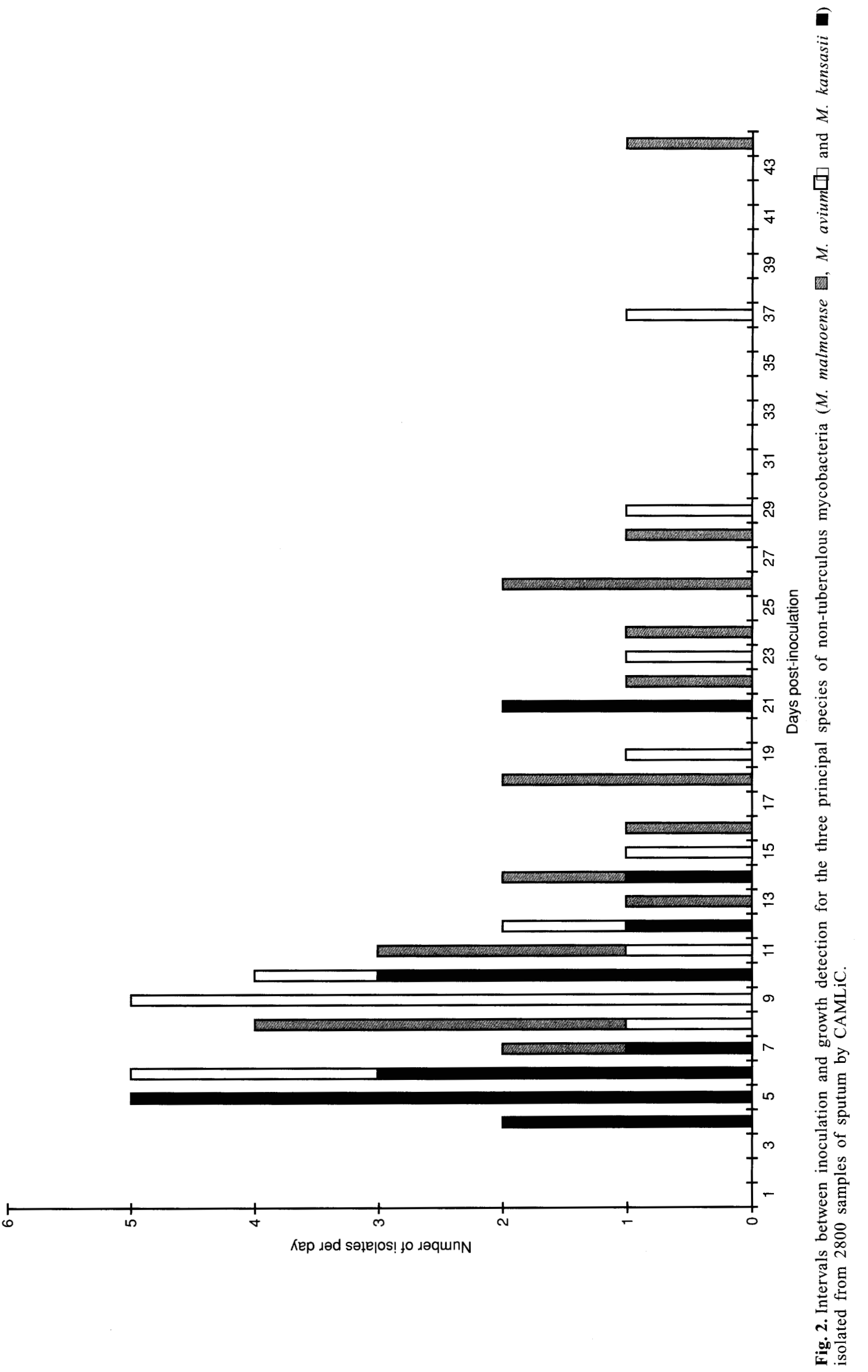




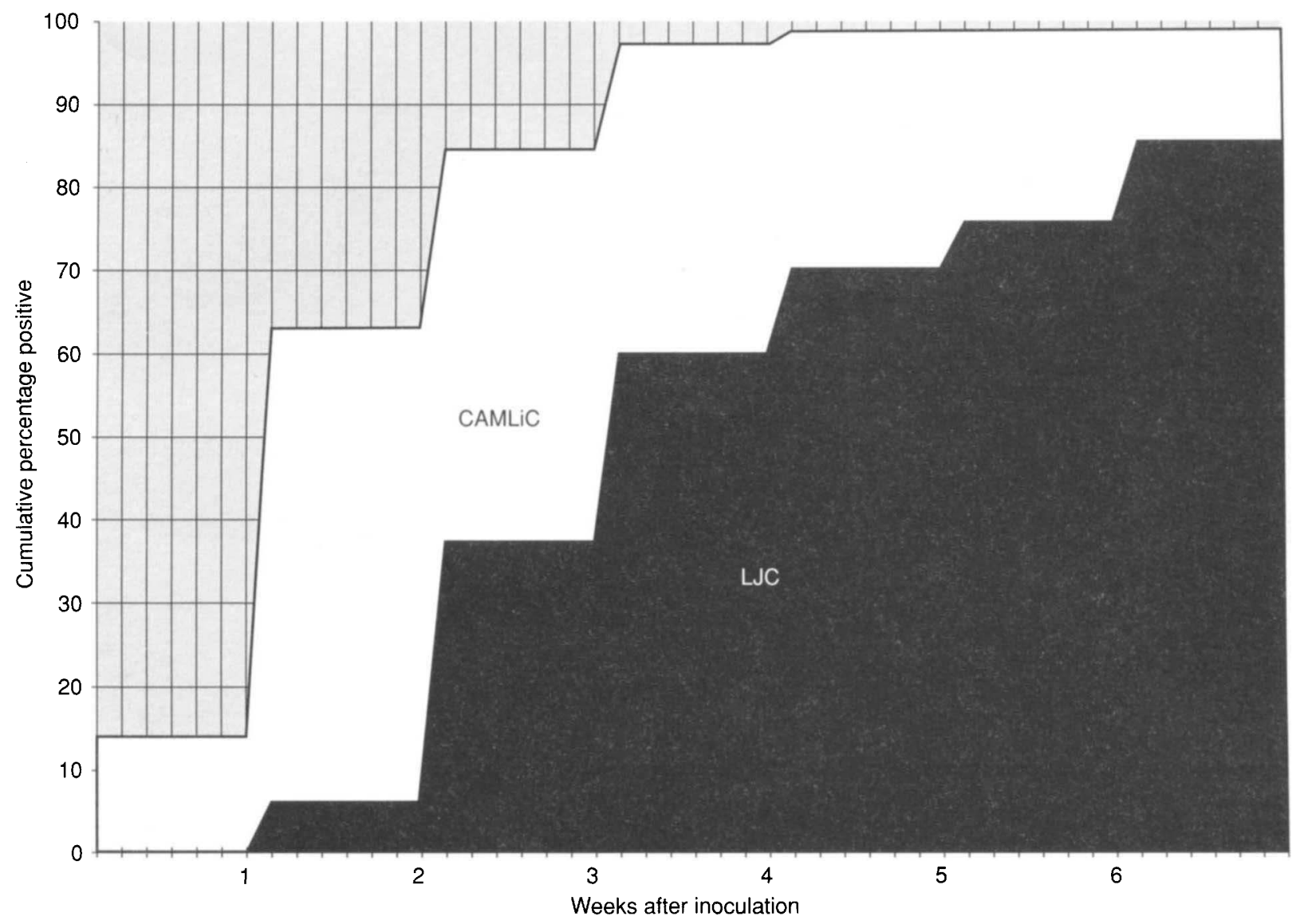

Fig. 3. Cumulative weekly positivity of CAMLiC and LJC from specimens yielding a total of 135 isolates of the $M$. tuberculosis complex by both methods combined.

Smear-negative samples take longer to become positive, but are still likely to be so within the first 30 days. The manufacturer recommends that specimens remain on the system for 6 weeks. The results of the present study suggest that the machine (which has a finite capacity) could be more efficiently used by discarding negative vials after 30 days, the vacated incubation slot being used for a fresh specimen, provided that conventional parallel LJC is continued for up to 9 weeks.

The enhanced speed of automated liquid culture also applies to contaminants. This means that most contaminated material is detected within a few days of inoculation, allowing re-processing of the specimen, re-inoculation of the system and re-institution of mycobacterial growth monitoring with only a short delay.

The CAMLiC approach is labour-sparing. After initial processing and inoculation, only positive vials require further attention, at least until discard. This laboratory has processed mycobacterial specimens daily throughout the normal working week for some years. We found it possible to introduce and use this technology without any increase in staffing or alteration to routine practices. The proportion of laboratories using CAM$\mathrm{LiC}$ systems able to achieve the $\mathrm{CDC}$ standard for detection and identification can be expected to be much higher than that reported for the SAMLiC system [2].

The net additional CAMLiC consumable cost per specimen was approximately $£ 3(\$ 5)$. Therefore, the additional cost for the 2800 specimens evaluated was $£ 8400$ (\$14000) when compared to conventional examination. To this must also be added the cost of c. 73 gene probes $(73 \times £ 12=£ 876 ; \$ 1400)$. If gene probes had been used on all 191 isolates the total cost for this would have been $£ 2300$ (\$3700), giving a maximum total of $£ 10700$ ( $\$ 17100$ ). However, this is the cost of applying CAMLiC indiscriminately to all specimens received. The equivalent figure for direct DNA detection on all 2800 specimens, equally distributed throughout the months of the study would be the cost of 150 tests per month. As the manufacturers' instructions dictate a kit (50 tests) shelf life of 1 months, three kits/month will be necessary ( $£ 3000$, approximately). This gives a total direct DNA detection cost of $£ 54000$ (\$86400).

If either method can be successfully targeted to a subset of specimens significantly more likely to be positive the cost-benefit ratio will become progressively more attractive. Taking this argument to an absurd conclusion, if the CAMLiC system was applied 
only to the smear-positive specimens $(c .80 \%$ of the culture positives in this laboratory and thus a calculated total of 153 specimens in this study), the net additional cost for the detection and identification of these mycobacteria to the $\mathrm{CDC}$ standard by CAMLiC would be only $£ 2300$ (\$3700), including gene probe identification for each isolate. Direct DNA detection techniques on the same 153 smear positive specimens (the currently licensed indication for these methods in the USA) would have necessitated one kit per month (assuming equal distribution throughout the study period), costing $£ 18000$ ( $\$ 28800$ ). In both cases $20 \%$ of the mycobacteria would have gone undetected, at least by a rapid technique, although the CAMLiC system would have provided cultures much earlier for susceptibility studies, the speed of which is another CDC standard to be attained [1].

Criteria based on patient characteristics (contacts of known cases, tuberculin skin test reactivity, chest Xray appearances, and so on) as well as specimen characteristics (smear positivity, for instance) might better define a subset of specimens larger than those that are simply smear positive, to receive routine CAMLiC examination. This would allow the achievement of the CDC standard for detection and identification for all mycobacteria-bearing specimens in the most cost-effective way. Identification of a subset of specimens most likely to contain mycobacteria will also provide the material for a direct comparison of CAMLiC, LJC and direct DNA detection techniques and lead to a full cost-benefit analysis of the place and appropriateness of these different technologies, which need not be mutually exclusive. Further work is required to determine such criteria. In the meantime it should be noted that investigations, particularly those that seek to assess the sensitivity and specificity of direct DNA detection methods and take culture of $M$. tuberculosis as the gold standard for comparison, must now specify the nature of the cultural method used, as automated liquid culture appears to be significantly more sensitive than LJC.

The evaluation reported here was with one specific version of the CAMLiC principle, but the conclusions will probably apply to any system which incorporates the three essential elements: (a) continuous growth monitoring, (b) a sufficiently sensitive growth detection method to bring detection within 14-21 days and (c) a culture system that provides sufficient biomass at the time of detection to permit valid direct gene probe examination. All such systems will permit laboratories to approach the CDC standards and currently represent the single most cost-effective manner of so doing. However, this report does not address the important issues of safety and laboratory containment which will inevitably accompany the widespread introduction of liquid mycobacterial culture techniques. Such matters, not least their costs, will also be important in deciding the optimum deployment of CAMLiC technology.

This work was supported in part by Organon Teknika Ltd. None of the authors has a commercial interest in Organon Teknika Ltd.

\section{References}

1. Tenover FC, Crawford JT, Huebner RE, Geiter LJ, Horsburgh CR, Good RC. The resurgence of tuberculosis: is your laboratory ready? J Clin Microbiol 1993; 31: 767-770.

2. Doern GV. Diagnostic mycobacteriology: Where are we today? $J$ Clin Microbiol 1996; 34: 1873-1876.

3. Vuorinen P, Miettinen A, Vuento R, Hällström O. Direct detection of Mycobacterium tuberculosis complex in respiratory specimens by Gene-Probe Amplified Mycobacterium Tuberculosis Direct Test and Roche Amplicor Mycobacterium Tuberculosis Test. $J$ Clin Microbiol 1995; 33: 1856-1859.

4. Styrt BA, Shinnick TM, Ridderhof JC, Crawford JT, Tenover FC. Turnaround times for mycobacterial cultures. $J$ Clin Microbiol 1997; 35: 1041-1042.

5. Huebner RE, Good RC, Tokars JI. Current practices in mycobacteriology: results of a survey of state public health laboratories. J Clin Microbiol 1993; 31: 771-775.

6. Bennedsen J, Larsen SO. Examination for tubercle bacilli by fluorescence microscopy. Scand J Resp Dis 1996; 47: 114-120.

7. Magee JG, Freeman R, Barrett A, Attrill H, Lightfoot NF. Testing for tuberculosis. Lancet 1996; 347: 476 . 